\title{
A missense point mutation (Ser515Phe) in the adrenoleukodystrophy gene in a family with adrenomyeloneuropathy: a clinical, biochemical, and genetic study
}

\author{
Matthias Vorgerd, Sigrid Fuchs, Martin Tegenthoff, Jean-Pierre Malin
}

\begin{abstract}
A 36 year old male patient with adrenomyeloneuropathy (AMN) developed progressive spastic paraparesis and sensory ataxia from the age of 18 . Biochemical studies showed increased plasma concentrations of saturated very long chain fatty acids (VLCFAs), subclinical evidence of adrenal insufficiency, and primary hypogonadism. Three female family members had increased plasma concentrations of VLCFAs, suggesting carrier status of adrenoleukodystrophy (ALD). Molecular genetic analysis detected a missense point mutation (C1930T) in exon 6 within the ALD gene, which predicts substitution of an amino acid (Ser515Phe) that is conserved between the deduced amino acid sequence of the peroxisomal membrane protein PMP70 and ALD protein. Detection of this point mutation allows diagnosis of ALD or AMN, identification of heterozygotes, and prenatal diagnosis of ALD.
\end{abstract}

(F Neurol Neurosurg Psychiatry 1995;58:229-231)

Keywords: adrenomyeloneuropathy; ALD gene; molecular genetic analysis

Adrenoleukodystrophy (ALD) is an $\mathrm{X}$ linked peroxisomal disease affecting $1 / 20000$ males either as cerebral ALD in childhood or as adrenomyeloneuropathy (AMN) in adulthood. ${ }^{3}$ Adrenomyeloneuropathy is a milder form of ALD with onset at 20-30 years of age and is clinically characterised by chronic progressive myelopathy with clinical manifestation of adrenal insufficiency. ${ }^{2}$ Heterozygotes of ALD or AMN may be clinically symptomatic. $^{3}$

The accumulation of very long chain fatty acids (VLCFAs) in the nervous system and adrenal gland due to impaired $\beta$-oxidation in the peroxisomes is the principal biochemical abnormality of ALD. ${ }^{7}$ Clinical diagnosis of ALD or AMN is supported by finding increased VLCFAs in plasma. ${ }^{3}$

Recently, a candidate gene for ALD was identified by positional cloning that was found to be partially deleted in six of 85 unrelated patients. ${ }^{4}$
We describe a German family in which a male patient with $\mathrm{AMN}$ and three women were possible heterozygotes. Molecular genetic analysis of the ALD gene allows diagnosis of ALD or AMN, carrier detection, and prenatal diagnosis.

\section{Case report}

CLINICAL AND INVESTIGATIVE FEATURES

A 36 year old man developed slowly progressive weakness of his legs, ataxia, and alopecia at the age of 18 (III.1, fig 1).

On examination he had spastic paraparesis, incomplete sensory level below L1 on both sides, and gait ataxia. There were no clinical signs of Addison's disease. Magnetic resonance imaging of the head and cervical, thoracic, and thoracolumbar region was normal. Plasma cortisol concentration was slightly decreased, plasma ACTH was increased, and an ACTH stimulation test was negative. Plasma testosterone was decreased and $\mathrm{LH}$ and $\mathrm{FSH}$ were raised.

The patient's maternal 83 year old grandmother (I.1, fig 1) developed slowly progressive spastic paraparesis at the age of 60 . She has had non-insulin dependent diabetes since the age of 70 . Neurological examination showed spastic paraparesis. Laboratory evaluation showed normal plasma concentrations of cortisol, ACTH, and a normal ACTH test.

The patient's 60 year old mother (II.2, fig 1) and his 25 year old sister (III.2, fig 1) had normal clinical examinations. Plasma cortisol and ACTH concentrations and an ACTH test were normal.

BIOCHEMICAL STUDIES

Plasma C22:0, C24:0, and C26:0 fatty acids were determined by liquid chromatography and compared with control samples. ${ }^{3}$

Plasma VLCFA concentrations (mean (SD)) in the patient with AMN and heterozygous family members

\begin{tabular}{|c|c|c|c|c|c|}
\hline & Controls & $\begin{array}{l}\text { atient wit } \\
A M M N\end{array}$ & I.1 & II. 2 & III. 2 \\
\hline ) & 1 & $17 \cdot 1$ & $12 \cdot 8$ & $7 \cdot 8$ & 3 \\
\hline 24 & 12. & 29.9 & 13.6 & 20.5 & $20 \cdot 2$ \\
\hline$/ \mathrm{ml})$ & $0.21(0.05)$ & $1 \cdot 12$ & 0.61 & 0.62 & 0.49 \\
\hline $22: 0$ & $0.81(0.1)$ & 1.7 & 1.07 & $1 \cdot 15$ & $1 \cdot 16$ \\
\hline $26: 0 / \mathrm{C} 22: 0$ & $0.013(0.00$ & 0.066 & 0.048 & 0.035 & 0.028 \\
\hline
\end{tabular}




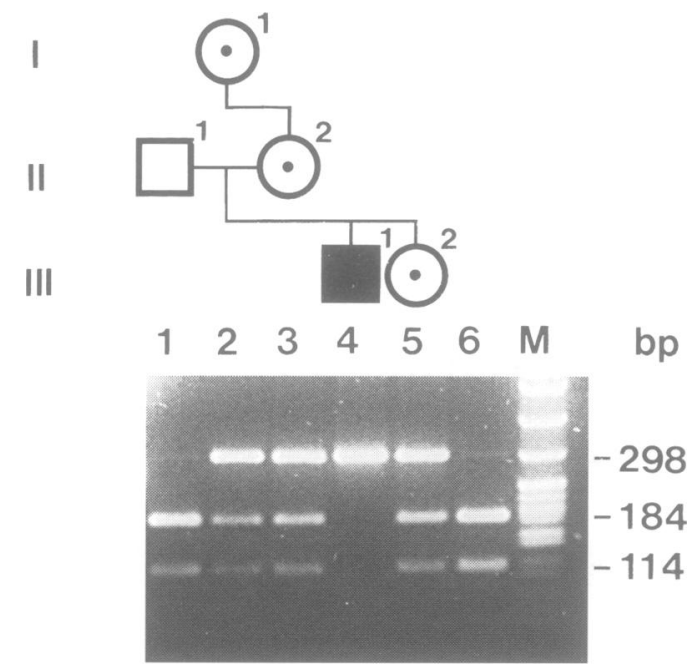

Figure 1 Identification of the C1930T (Ser515Phe) mutation by SacI digestion in the German kindred with AMN. - affected, $\odot$ heterozygote; lanes 1 and 6 , wild type pattern of the patient's unaffected father (II.1) and an unrelated control respectively; lane 4, hemizygous loss of an SacI site due to the C1930T transition (Ser515Phe) in the DNA of the patient with AMN (III.1); lanes 2, 3, and 5 , heterozygous SacI restriction pattern of obligate carriers I.2, II.2, and III.2, respectively; $M$, molecular weight standard ( $p B R 322 / M s p I)$. Fragment lengths (bp) are on the right.

In the patient plasma VLCFAs (C26:0) were increased fivefold and the plasma VLCFA ratio C26:0/C22:0 was also increased fivefold compared with values for controls. Plasma C26:0 VLCFAs and C26:0/C22:0 VLCFA ratios were increased twofold in the patient's sister (III.2) and threefold in the mother (II.2) and maternal grandmother (I.1).

\section{MOLECULAR GENETIC ANALYSIS}

Genomic DNA of the patient with AMN presented in this study was analysed for possible sequence variations in the ALD gene by polymerase chain reaction (PCR) amplification and single strand conformation polymorphism (SSCP) analysis. ${ }^{5}$ The PCR segment

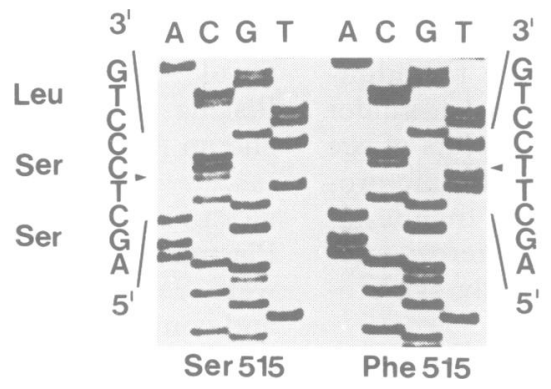

Leu

Phe

Figure 2 Detection of the C1930T (Ser515Phe) mutation by direct sequencing of $P C R$ products containing exon 6 of the ALD gene. Products of an asymmetric PCR with one primer diluted to 1:50 was sequenced using the diluted primer in the chain termination method (Sequenase kit, USB) essentially according to the instructions of the manufacturer. ${ }^{\prime}$ The wild type sequence is on the left, the mutant one of the patient with AMN on the right. The arrowheads point to the substituted nucleotide. containing exon 6 of the ALD gene showed a clearly aberrant SSCP pattern (data not shown). Direct sequencing of this particular segment showed a single nucleotide substitution (C1930T) in the patient's sequence (fig 2), predicting the replacement of serine to phenylalanine at position 515 (Ser515Phe) in the deduced amino acid sequence of the ALD gene (numbering of nucleotides and amino acids to the ALD cDNA sequence as in Mosser et $\mathrm{al}^{4}$ ). This C1930T transition destroys a recognition site for the restriction endonuclease SacI in the ALD sequence and can therefore easily be detected by restriction enzyme analysis both in relatives of the patient and in unrelated controls (fig 1). SacI digestion of the wild type 298 base pair (bp) PCR product generates two fragments of 184 and $114 \mathrm{bp}$, whereas the PCR product carrying the $\mathrm{C}$ to $\mathrm{T}$ substitution is not cleaved. As shown in fig 1 the Ser515Phe mutation could also be detected in a heterozygous manner in the three female relatives of the patient-who also showed increased VLCFA concentrations-but not in the unaffected father. Restriction enzyme analysis of DNA samples from unaffected controls showed that this sequence mutation was not present in 60 unrelated $\mathrm{X}$ chromosomes (data not shown).

\section{Discussion}

Adrenoleukodystrophy is an X linked disease characterised by abnormal myelin accumulation of VLCFAs due to impaired $\beta$-oxidation in the peroxisomes. ${ }^{7}$ Different clinical phenotypes coexist within the same kindred. ${ }^{23}$ Childhood ALD is the more severe form with onset of neurological symptoms between 5 and 12 years of age. Demyelination in the CNS progresses rapidly and leads to death within a few years. ${ }^{3}$ The milder AMN form affects the spinal cord and peripheral nerves with onset at 20-30 years of age and a more progressive course. ${ }^{23}$

We presented a patient with clinical and biochemical evidence of AMN who developed progressive spastic paraparesis and sensory ataxia. Diagnosis was confirmed by greatly increased plasma VLCFAs.

Neuroradiological studies in patients with AMN show white matter alterations on brain MRI in nearly half of the patients and most patients show atrophy of the thoracic cord. ${ }^{3}$ Magnetic resonance imaging of the neuraxis was, however, normal in our patient.

According to Moser and Moser, most of the patients with ALD or AMN have clinical or biochemical evidence of impaired adrenal function or reserves. ${ }^{3}$ Adrenal function tests in our patient suggested adrenal insufficiency without clinical signs or symptoms of Addison's disease. The patient's primary hypogonadism could be due to damage of the testis in the course of the peroxisomal disease. $^{6}$

Ten to fifteen per cent of women heterozygous for ALD or AMN develop progressive myelopathy and peripheral neuropathy, usually manifesting for the first time in their fifth 
decade. Adrenal function is almost always intact in carriers of the disease. ${ }^{3}$

Three women in the pedigree presented were shown to have increased plasma VLCFAs, suggesting ALD or AMN heterozygotes. The patient's maternal grandmother, who presented with the clinical picture of $A M N$ of late onset, could therefore be a symptomatic ALD heterozygote.

Recently, the identification of a candidate gene for ALD on the distal arm of the X chromosome was reported. ${ }^{4}$ The deduced amino acid sequence of the ALD protein shows significant homology $(75 \%)$ to a 70 $\mathrm{kDa}$ peroxisomal membrane protein PMP70. ${ }^{8}$ Both ALD protein and PMP70 share consensus sequences for ATP binding folds at the $C$ terminal part of the protein that attaches them to the ATP binding cassette (ABC) family of transporters, which are involved in the transport of amino acids, inorganic ions, peptides, and proteins. ${ }^{48}$ Immunofluorescence analysis and immunoelectron microscopy have shown that the ALD protein is indeed located in the peroxisomal membrane, but the putative substrate of the ALD protein remains unknown. ${ }^{9}$

The ALD gene was partially deleted in six out of 85 independent ALD patients. ${ }^{4}$ In some cases missense point mutations in the ALD gene of patients have been identified. ${ }^{110}$ Molecular genetic analysis of the genomic DNA of the patient with AMN presented in this study detected a missense point mutation (C1930T) in exon 6 of the ALD gene that predicts the change of serine to phenylalanine at position 515 (Ser515Phe) in the deduced amino acid sequence. Because Ser 515 is present in one of the ATP binding folds that is entirely conserved between the ALD protein and PMP70, this substitution might disturb the proper binding of ATP and consequently impair the function of the protein. ${ }^{11}$

If the Ser515Phe substitution found in the family was the only mutation present in the ALD gene, as our results suggest, it could be the likely cause of the disease. Additional examinations, such as in vitro expression studies, may provide a final proof. Nevertheless, as the nucleotide change does not seem to be a common polymorphism, and in the family cosegregates with the disease phenotype, it can be used for carrier and prenatal diagnostic tests in the patient's blood relatives.

Analysis of DNA of the patient's mother, maternal grandmother, and sister showed that they carry the ALD mutation Ser515Phe in a heterozygous manner. Therefore, the 25 year old sister has a $50 \%$ chance of transmitting the mutated ALD gene to her offspring.

Identification of the Ser515Phe mutation principally allows prenatal diagnosis of ALD or AMN. It is not possible to predict the detailed clinical phenotype, however, because the same mutation can cause ALD or AMN within the same family. ${ }^{410}$ Additional factors presumably play a part in determining the final phenotype.

1 Cartier N, Sarde CO, Douar AM, Mosser J, Mandel JL, Aubourg P. Abnormal messenger RNA expression and a missense mutation in patients with X-linked adrenoleukodystrophy. Hum Mol Genet 1993;2: 1949-51.

2 Griffin JW, Goren E, Schaumburg H. Adrenomyeloneuropathy: a probable variant of adrenoleukodystrophy. Clinical and endocrinological aspects. Neurology 1977;27:1107-13.

3 Moser HW, Moser A. Adrenoleukodystrophy (X-linked) In: Scriver CR, Beaudet AL, Sly WS, eds. The metabolic basis of inherited disease. 6th ed. New York: McGrawbasis of inherited diseas

4 Mosser J, Douar AM, Sarde CO. Putative X-linked adrenoleukodystrophy gene shares unexpected homology with ABC transporters. Nature 1993;361:726-30.

5 Orita M, Suzuki Y, Sekyia T, Hayashi K. Rapid and sensitive detection of point mutations and DNA polymorphisms using the polymerase chain reaction. Genomics 1989;5:874-9.

6 Powers JM. The testis in adrenoleukodystrophy. $A m f$ Pathol 1981;102:90-8.

7 Singh I, Moser HW, Moser A. Adrenoleukodystrophy: impaired oxidation of very long chain fatty acids in cultured skin fibroblasts and

8 Kamijo K, Taketani S, Yokota S, Osumi T, Hashimoto T The $70 \mathrm{kDa}$ peroxisomal membrane protein is a member of the Mdr (P-glycoprotein)-related ATP-binding ber of the Mdr (P-glycoprotein)-related ATP-binding
protein superfamily. $\mathscr{f}$ Biol Chem 1990;265:4534-40.

9 Mosser J, Lutz Y, Stoeckel ME, Sarde CO, Kretz C, Douar AM, Lopez J, Aubourg P, Mandel JL. The gene responsible for adrenoleukodystrophy encodes a peroxisomal membrane protein. Hum Mol Genet 1994;3: 265-71.

10 Fuchs S, Sarde CO, Wedemann H, Schwinger E, Mandel $\mathrm{JL}, \mathrm{Gal} \mathrm{A}$. Missense mutations are frequent in the gene for X-chromosomal adrenoleukodystrophy. Hum Mol Genet 1995 (in press)

11 Gyllenstein UB, Erlich HA. Generation of single-stranded DNA by polymerase chain reaction and its amplification to direct sequencing of the HLA-DQA locus. Proc Natl Acad Sci USA 1988;85:7652-6. 